\title{
A blood pool contrast aided T1 functional MRI in patients with brain tumors-a preliminary study
}

\author{
Agata Majos • Tomasz Wolak • Piotr Bogorodzki • \\ Krzysztof Tybor $\cdot$ Michal Sapieha $\cdot$ Ludomir Stefańczyk
}

Received: 14 July 2010 /Accepted: 17 November 2010 /Published online: 17 December 2010

(C) The Author(s) 2010. This article is published with open access at Springerlink.com

\begin{abstract}
Introduction The aim of our study was to determine the possibility of using a new functional technique: a T1dependent sequence with administration of blood pool contrast agent (BPCA), in patients with brain tumors before and after surgical treatment. We also aimed to compare our results with those obtained using the fMRI technique, based on Blood Oxygenation Level-Dependent (BOLD) contrast. Methods For each of our 14 oncologic patients (four before and ten after neurosurgical intervention), we obtained: a T1$3 \mathrm{D}$ GRE sequence $\left(\mathrm{TR}=2.6 \mathrm{~ms} / \mathrm{TE}=1.1 \mathrm{~ms} / \mathrm{FA}=10^{\circ}\right)$ after intravenous administration of BPCA $(0.03 \mathrm{mmol} / \mathrm{kg})$, as well as a $\mathrm{T} 2 * \mathrm{EPI}$ sequence $\left(\mathrm{TR}=3 \mathrm{~s} / \mathrm{TE}=50 \mathrm{~ms} / \mathrm{FA}=90^{\circ}\right)$. Movement and/or tactile block type paradigms were carried out during both functional runs. SPM5 software was used for analysis.

Results For both functional techniques, maximum activations were localized in the same areas. There were no significant differences observed in the $t$ values calculated for activations located in the primary motor cortex between groups of pre- and post-intervention patients (in the same
\end{abstract}

\footnotetext{
A. Majos $(\varangle) \cdot$ M. Sapieha $\cdot$ L. Stefańczyk

Radiology Department, Medical University of Lodz,

Poland 90-153, Lodz Kopcinskiego 22

e-mail: agata.majos@umed.lodz.pl

T. Wolak

Institute of Physiology and Pathology of Hearing,

Poland 01-943, Warsaw Pstrowskiego 1
}

\section{P. Bogorodzki}

Institute of Radioelectronics, Warsaw University of Technology,

Poland 00-665, Warsaw Nowowiejska 15/19

K. Tybor

Neurosurgery Department, Medical University of Lodz,

Poland 90-153, Lodz Kopcinskiego 22 functional technique). The mean values for T2* EPI examinations were 10.84 and 9.36 , respectively. The mean $t$ values for the T1 technique were lower, especially for the post-intervention patients (5.83 and 3.9, respectively).

Conclusions The $\mathrm{T} 1$ technique can be used to detect functional areas in patients with brain tumors, pre-, and post-surgical intervention. This technique enables the evaluation of cortical centers that suffer from susceptibility artifacts when using the T2* BOLD technique. Activations found using both techniques have the same localization, with lower values for the $\mathrm{T} 1$ technique.

Keywords Functional MRI C CBV . Contrast media $\cdot$ Brain tumors $\cdot$ Neurosurgery

\section{Introduction}

The Blood Oxygenation Level-Dependent (BOLD) effectbased technique has for years been the most significant and valuable technique used during the examination of cerebral center activity. As for every technique, however, it has some limitation [1-4]. The T2*-weighted echo planar (EPI) sequences, being the basis for BOLD contrast detection, are characterized with high sensitivity to magnetic field heterogeneity. Therefore, adjacent areas that differ significantly in terms of magnetic susceptibility may be examined with $\mathrm{AMRI}$ BOLD only provided that specific techniques are employed [5-7]. If such techniques are not used, examination of these areas will not be possible due to the presence of marked artifacts or signal loss. Basal parts of the frontal lobes, connecting to the paranasal sinuses, as well as the basal parts of the temporal lobes (which are located in close proximity to the compact parts of the petrous bones) represent important regions of the brain 
which are particularly difficult to examine using functional fMRI BOLD evaluation. Examination of brain regions located next to clinical lesions and/or post-surgical artifacts such as scars, vascular clips, stent grafts, or CranioFixes is another important clinical application of functional studies. These studies are performed in patients after neurosurgeries, and particularly in subjects who were operated on due to brain tumors and are followed up in context of the relapse $[4,8]$.

The cerebral blood volume (CBV) technique, based on T1-weighted sequences, constitutes an alternative to $\mathrm{FMRI}$ BOLD used in the examination of cerebral center activity. This method utilizes T1-dependent sequences. The advantages of this novel technique which are of special meaning are: better signal to noise ratio, smaller artifacts due to large venous vessels, high spatial resolution, and more localized and at the same time highly specific mapping of the brain activations [9-12]. Clinical applications of novel contrast agents, namely blood pool contrast agents (BPCA), enable the use of $\mathrm{CBV}$ in the functional evaluation of healthy volunteers and patients [11, 13-18]. However, no studies have been performed thus far describing the usefulness of this technique in the localization of active cerebral centers in neurosurgical patients.

The aim of this study was to verify the usefulness of the sensitive T1-weighted sequence along with blood pool contrast agents, when establishing functional maps of eloquent cortical regions in brain tumor patients before and after neurosurgeries. Moreover, the efficacy of the aforementioned method was compared to the standard functional MRI technique based on BOLD contrast.

\section{Materials and methods}

Four patients (14-58 years of age; mean age of 25.7; one female, three males), with a diagnosis of central nervous system tumors based on prior computer tomography and MRI examinations were included in the first group. All of these subjects were qualified for surgical treatment and subsequently underwent surgical interventions.

The second group consisted of ten patients (23-58 years of age; mean age of 42.2; four females, six males) who had previously undergone neurosurgical interventions for brain tumors; in five cases, the subjects had been diagnosed with recurrent tumors. Of the ten patients, seven had previously undergone one surgical operation, two had undergone two interventions, and one had undergone three interventions. The patients in this group underwent the following surgical procedures: one had undergone a partial resection, three had had a subtotal resection, with the remaining six patients having undergone total resections of the tumors. All the procedures were performed from 3 days to 91 months prior to the functional examinations included into this study. Detailed data is presented in Table 1.

The ethics committee at our institution approved these procedures, which were conducted in accordance to the principles established by the Helsinki Convention. Informed consent was obtained from all participants.

Examinations were performed using a 1.5-T scanner, Avanto, Syngo ver. N4VB13A with 12ch HeadMatrix coil, Siemens, Germany. Both EPI sequences, as well as T1 spin echo (SE) were performed using standard parameters routinely used in clinical practice. First, a high-resolution T1 anatomical scan was performed with $1 \times 1 \times 1 \mathrm{~mm}$ isotropic voxels. A three-dimensional FLASH sequence was obtained according to the following protocol: $\mathrm{FOV}=$ $256 \times 256 \mathrm{~mm}$, matrix $=256 \times 256, \mathrm{TR}=8.8 \mathrm{~ms}, \mathrm{TE}=4.8 \mathrm{~ms}$, $\mathrm{FA}=25^{\circ}, \mathrm{TA}=5^{\prime} 07$. Each volume acquired contained 160 slices, $1 \mathrm{~mm}$ thick, with no gap. Next, a reference scan (run 1) was acquired with a typical T2*-sensitive GRE EPI sequence: $\mathrm{TR}=3000 \mathrm{~ms}, \mathrm{TE}=50 \mathrm{~ms}, \mathrm{FA}=90 \mathrm{deg}, \mathrm{FOV}=$ $240 \times 240 \mathrm{~mm}$, iPAT $=2$, pixel bandwidth $=2605 \mathrm{~Hz} / \mathrm{Px}$, for $383-\mathrm{mm}$ thick slices with $0.75 \mathrm{~mm}$ gap and $3.75 \mathrm{~mm}$ inplane resolution. All slices where positioned in the axial plane parallel to the AC-PC line. One hundred volumes were acquired with total $\mathrm{TA}=$ 5'12 (four dummy scans).

BPCA was administrated in the second part of the examinations. MS-325, at the time of our study produced as Vasovist $^{\mathrm{TM}}$ (Bayer Schering Pharma, Berlin), now known as Ablavar $^{\mathrm{TM}}$ (Lantheus Medical Group), is a contrast agent containing a chelate of gadolinium diethylenetriaminepentaacetato with a (diphenylcyclohexyl) phosphono-oxymethyl substituent (Gadofosveset Trisodium) at a concentration of $0.25 \mathrm{mmol} / \mathrm{ml}$. Following intravenous administration, it reversibly binds to albumin plasma protein $(80-87 \%)$, thus extending plasma half-life $\left(0.48 \pm 0.1 \mathrm{~h}\right.$ for $\mathrm{t} \_1 / 2$ alpha-halflife for distribution phase, and $\sim 16 \mathrm{~h}$ for $\mathrm{t} \_1 / 2$ _beta-half-life for the elimination phase) and limiting its movement out of blood vessels. The relaxation value of the plasma at $20 \mathrm{MHz}$ is calculated between 33.4 and $45.7 \mathrm{mM}^{-1} \mathrm{~s}^{-1}$ for doses of $0.05 \mathrm{mmol} / \mathrm{kg} \mathrm{mc}$ [19-21]. Both these characteristics underline the value of MS-325 for fMRI with T1dependent sequences [22]. Also, MS-325 might enable the use of a new approach in imaging mild or non-enhancing tumors using conventional extracellular contrast agents [23]. It was administered in doses recommended by the manufacturer for standard examinations: MR angiography of abdominal and lower extremity vessels - i.e., $0.12 \mathrm{ml} / \mathrm{kg}$ b.w., which is equal to $0.03 \mathrm{mmol} / \mathrm{kg}$ b.w.

After intravenous administration of the contrast medium, a second T1-sensitive (run2) scan was performed using a 3D GRE sequence according to the following protocol: $\mathrm{FOV}=228 \times 228 \mathrm{~mm}$, matrix $=128 \times 128, \mathrm{TR}=2.6 \mathrm{~ms}, \mathrm{TE}=$ $1.1 \mathrm{~ms}, \mathrm{FA}=10^{\circ}$, iPAT $=2$, bandwidth $=1185 \mathrm{~Hz} / \mathrm{Px}$, inplane resolution $=1.78 \times 1.78 \mathrm{~mm}$. Forty slices in the axial 
Table 1 Patients' data regarding localization, pathological type of the tumor, and kind of used neurosurgical procedure
AG Astrocytoma gemistocyticum, PNET primitive neuroectodermal tumor, $M$ meningioma, AF Astrocytoma fibrillare, $O D G$ Oligodendroglioma, OAA Oligoastrocytoma anaplasticum, HAP Haemangiopericytoma-like tumor, $F$ frontal lobe, $T$ temporal lobe, $P$ parietal lobe, $O$ occipital lobe

\begin{tabular}{llllll}
\hline No & Initials & Localization & Tumor type & Time between MRI and surgery in months & surgery \\
\hline \multicolumn{2}{l}{ MRI exam before surgery } & & & \\
1 & KZ & P/T left & AG & 1 & Subtotal \\
2 & MP & F right & PNET & 2 & Biopsy \\
3 & KW & F left & PNET & 1 & Biopsy \\
MRI exam after & surgery & & & \\
4 & EF & F/T left & M & 10 & Total \\
5 & RC & T left & AF/GM & 91 & Partial \\
6 & ZN & P/O right & meta colon & 3 days & Subtotal \\
7 & MF & P left & AF/GM & 1 & Subtotal \\
8 & WC & F left & ODG & 3 days & Total \\
9 & EW & F right & AF & 100 & Total \\
10 & AS & P left & OAA & 10 & Subtotal \\
11 & MK & F right & HAP & 68 & Total \\
12 & JS & F left & AF & 11 & Total \\
13 & BC & F left & AF & 10 & Total \\
\hline
\end{tabular}

plane where acquired, each 3-mm thick with a $0.5 \mathrm{~mm}$ gap. One hundred and five volumes were acquired in TA=10'30. The first five volumes were rejected so as to achieve stable magnetization.

During the same session, a more comprehensive morphology protocol was performed on 11 patients in order to bring their radiological diagnoses up to date. For this reason, functional $\mathrm{CBV}$ examinations were started immediately or up to $10 \mathrm{~min}$ after contrast agent administration. The total time of each session did not exceed $60 \mathrm{~min}$.

All subjects were trained to rehearse the paradigm task a few minutes before the procedure. Each patient performed between one and three paradigms. In cases where the pathological mass was located near the central sulcus, the finger tapping paradigm was used. In two of these cases, touch stimuli were applied. The movement task required the patient to freely move the fingers of his or her contralateral hand in relation to the hemisphere with the tumor or postsurgical lesions. Depending on the patient's neurological state, the movement they had to perform was either to touch their thumb sequentially with fingers II, III, IV, and V, or to clench and open their fist. In cases where touch stimuli were used, the dorsum of the hand was stroked by an experimenter using a coarse brush. For both the finger tapping and the touch stimuli methods, each hand was examined separately in order to evaluate secondary and additional centers of activation.

Preprocessing Data was analyzed using statistical parametric mapping (SPM5) software (Wellcome Department of Imaging Neuroscience, University College London, London, UK). Movement correction was performed as the first preprocessing step for the BOLD and T1 CBV image series. All patients, with movements greater than
$2 \mathrm{~mm}$ or correlations with stimulating paradigms bigger than 0.5 , were excluded from the study. Subsequently, co-registration of T1 and EPI was performed in order to move two series in the same space and allow localization of activated regions. Spatial smoothing of functional images (Gauss smoothing filter with kernel FWHM= $7 \mathrm{~mm}$ ) was used to compensate for residual distortion between-subject variability after spatial normalization. A 128-s high-pass filter was applied to remove lowfrequency fluctuations in the BOLD response.

Modeling General linear modeling was used to set up a model and to fit it to the acquired data. The model timing was derived from individual subject performance logs.

Statistics Hot spot analyses were performed for individual patients. For each voxel, a $T$ value was calculated. Each voxel with a $T$ score above the threshold value was treated as an active voxel, with the condition that within the nearest vicinity were at least four "active voxels."

\section{Results}

In both groups: for patients who had previously undergone surgical procedures and those who had not, we observed an increase in signal activity in the primary sensorimotor cortex during both the BOLD contrast ( - in $85.7 \%$ and $100 \%$ of tasks, respectively) and the T1 CBV techniques (78.6\% and $85.7 \%$ of tasks).

We found a high concordance in the localization of activations between the two techniques. In the group of patients before surgical intervention, this was confirmed in 
all patients, in six out of seven tasks; in one case, massive movement artifacts were observed during T1 CBV examination. In the post-surgical intervention group, we found agreement in localization for nine out of the 14 performed tasks. In two cases, signal loss occurred during the EPI sequence due to the presence of postoperative materials, which made it impossible to obtain any results using this method. In one case, movement artifacts interfered with the interpretation of the T1 CBV examination, and two patients did not show statistically significant activations in one of their tasks (Table 2, Figs. 1, 2).

Using the BOLD technique, we found typical $t$ values in the activated areas ranging between 5.8 and 15. After surgery, these remained similar, except in one subject who failed to show activation at all. By comparison, using a blood pool contrast agent and T1-weighted images, we found $t$ values between 3 and 9.4 before surgery, somewhat reduced after surgery.

There were no significant differences in the $t$ values for the T2* EPI examinations between the pre- and postintervention patients in the same functional technique; the mean value for acquired data was (after excluding tasks with values $=0$ because of artifacts) 10.84 and 9.36, respectively. For the T1 CBV technique, the mean $t$ value for the post-intervention patients was lower: 5.83 and 3.9, respectively. However, this was the result of no activation during the single tasks of two patients. None of the patients demonstrated a lack of activation in the sensorimotor cortex for all of the tasks.

We observed activation of the cerebellum in four patients for six of the tasks using the BOLD technique. For the T1 CBV technique, activation in this area was observed in two of the same patients for three tasks. The mean $t$ values for the BOLD and T1 CBV techniques were 8.3 and 3.9, respectively. For the pre-intervention group, no areas of activation were observed in the cerebellum for any of the patients using the different methods (Fig. 3).

\section{Discussion}

Since the possibility of applying the T1 CBV technique was previously demonstrated during laboratory studies and in healthy volunteers [16], a control group was not included in this study. Although their cohort was small, Ben Bashat et al. [17] achieved satisfactory results during CBV evaluation of brain tumor patients using routinely applied gadolinium chelates with a mean distribution half-life of about $0.2 \mathrm{~h}$ (www.imaging.bayerhealthcare.com/html/magnevist).

The results of our study confirmed the high value of the tested procedure in patients qualified for neurosurgeries, particularly in those who were already subjected to surgical treatment. We observed increased signal intensity of the primary motor cortex (PMC) in four out of five paradigms applied to all three non-operated subjects, as well as in 12 out of 14 tasks performed by nine patients who were subjected to neurosurgical intervention. When using the T2* BOLD technique, an established and reliable source of functional information, during the same session, the activations were observed in all paradigms applied in preoperative studies and in 12 out of 14 tasks performed by all ten subjects who were examined postoperatively. The lack of cortical center activation in $\mathrm{T} 1 \mathrm{CBV}$ sequences was related to the presence of significant movement artifacts. In T2* EPI sequences, the activation was not noted due to signal loss caused by the presence of post-surgical materials.

The possibility of examining patients who were previously subjected to neurosurgical procedures seems to be the vital objective of T1 CBV technique application. Patients in whom tumor relapse was suspected were frequently disqualified from functional studies up to now. Using the BOLD technique, fibrous or metallic structures left after surgery disturbed or even disabled precise mapping of surrounding cortical centers located in the region of potential relapse. In this study, such a situation occurred in two out of ten patients examined. In echo planar sequences, signal loss was observed around the eloquent centers of these patients due to CranioFixes placed in their skulls. Significant, precisely located activations were observed in these patients, however, when the T1 CBV technique was applied (Fig. 4).

The presence of head movement artifacts in $\mathrm{T} 2 *$ BOLD examinations, observed also in our results, characterizes multi-shot techniques such as GRE. These artifacts, especially task-related, can influence significantly the examined cortex activities or/and create false negative results. One of this important problem solutions is to use single shot EPI by adding additional inversion pulse in EPI sequence what gives additional T1 weighting to $T^{*} 2$ EPI images $[24,25]$. A common application of Magnetization Prepared Rapid Gradient Echo (MPRAGE) is to provide precise anatomical references free from the distortions and dropouts of the fast, lowresolution, $\mathrm{T}^{*} 2$-weighted images used to obtain functional data. But one of our primary goals of this work was to use high-quality structural brain MP-RAGE sequences for functional imaging. A use of MP-RAGE also for functional imaging overcomes several problems associated with image co-registration and different $\mathrm{T}^{*} 2$ weighting specific to EPI sequence. We tuned our MP-RAGE sequence with respect to two parameters: brain coverage and volume acquisition time. Further studies with combination of EPI with inversion pulse in order to compare performance either IR-EPI or MP-RAGE are necessary but are beyond the scope of this paper. 
Table 2 Values of T-statistics in PMC and CBL in used paradigms both in EPI BOLD and CBV T1 methods

\begin{tabular}{|c|c|c|c|c|c|c|}
\hline \multirow[t]{3}{*}{ No } & \multirow[t]{3}{*}{ Initials } & \multirow[t]{3}{*}{ Paradigm } & \multicolumn{3}{|c|}{$T$ statistic value } & \\
\hline & & & \multicolumn{2}{|l|}{ EPI BOLD } & \multicolumn{2}{|l|}{ CBV T1 } \\
\hline & & & $\mathrm{PMC}$ & CBL ipsi/contr & PMC & CBL ipsi/contr \\
\hline \multicolumn{7}{|c|}{ MRI exam before surgery } \\
\hline 1 & $\mathrm{KZ}$ & right $\mathrm{H}$ movement & 15.14 & & 4.13 & \\
\hline \multirow[t]{2}{*}{2} & MP & left $\mathrm{H}$ movement & 5.81 & & 5.67 & \\
\hline & & right $\mathrm{H}$ movement & 8.55 & & 2.97 & \\
\hline \multirow[t]{2}{*}{3} & KW & right $\mathrm{H}$ movement & 9.23 & & 9.41 & \\
\hline & & left $\mathrm{H}$ movement & 8.9 & & Massive artifacts & \\
\hline \multicolumn{7}{|c|}{ MRI exam before surgery } \\
\hline 4 & $\mathrm{EF}$ & right $\mathrm{H}$ movement & 11.24 & & 4.28 & \\
\hline 5 & $\mathrm{RC}$ & right $\mathrm{H}$ movement & 7.36 & & 5.73 & \\
\hline 6 & $\mathrm{ZN}$ & right $\mathrm{H}$ movement & 9.96 & & Massive artifacts & \\
\hline \multirow[t]{2}{*}{7} & MF & right $\mathrm{H}$ movement & 8.84 & 8.83 & 4.45 & 3.43 \\
\hline & & right $\mathrm{H}$ sensory task & 3.66 & 7.68 & 2.25 & 5.07 \\
\hline 8 & WC & right $\mathrm{H}$ movement & 6.17 & 5.69 & 3.94 & \\
\hline 9 & EW & left $\mathrm{H}$ movement & 0 signal loss & & 4.99 & \\
\hline \multirow[t]{2}{*}{10} & AS & right $\mathrm{H}$ movement & 12.55 & 7.42 & 0 & \\
\hline & & right $\mathrm{H}$ sensory task & 3.47 & & 3.55 & \\
\hline 11 & MK & left $\mathrm{H}$ movement & 7.49 & & 5.36 & \\
\hline \multirow[t]{2}{*}{12} & JS & right $\mathrm{H}$ movement & 12.55 & 11.74 & 5.18 & 3.27 \\
\hline & & left $\mathrm{H}$ movement & 15.37 & $5.39 / 11.39$ & 0 & \\
\hline \multirow[t]{2}{*}{13} & $\mathrm{BC}$ & right $\mathrm{H}$ movement & 0 signal loss & & 5.57 & \\
\hline & & left $\mathrm{H}$ movement & 13.22 & & 5.6 & \\
\hline
\end{tabular}

$H$ hand

The motion artifacts present only in the CBV T1 may be due to several factors. EPI sequences were used relatively early, during the first phase of RM examination, prior to contrast agent application. The T1 CBV sequence was always the last one used in every patient. Since more than one paradigm was applied in four out of ten patients, and morphological sequences were used in all subjects also following contrast agent application, at the end of the study, patients were likely tired and less concentrated.

Fig. 1 Patient KW before surgery with tumor in the left frontal lobe; activations in PMC in T1 CBV technique upper row, in BOLD technique in lower row during right-hand movement

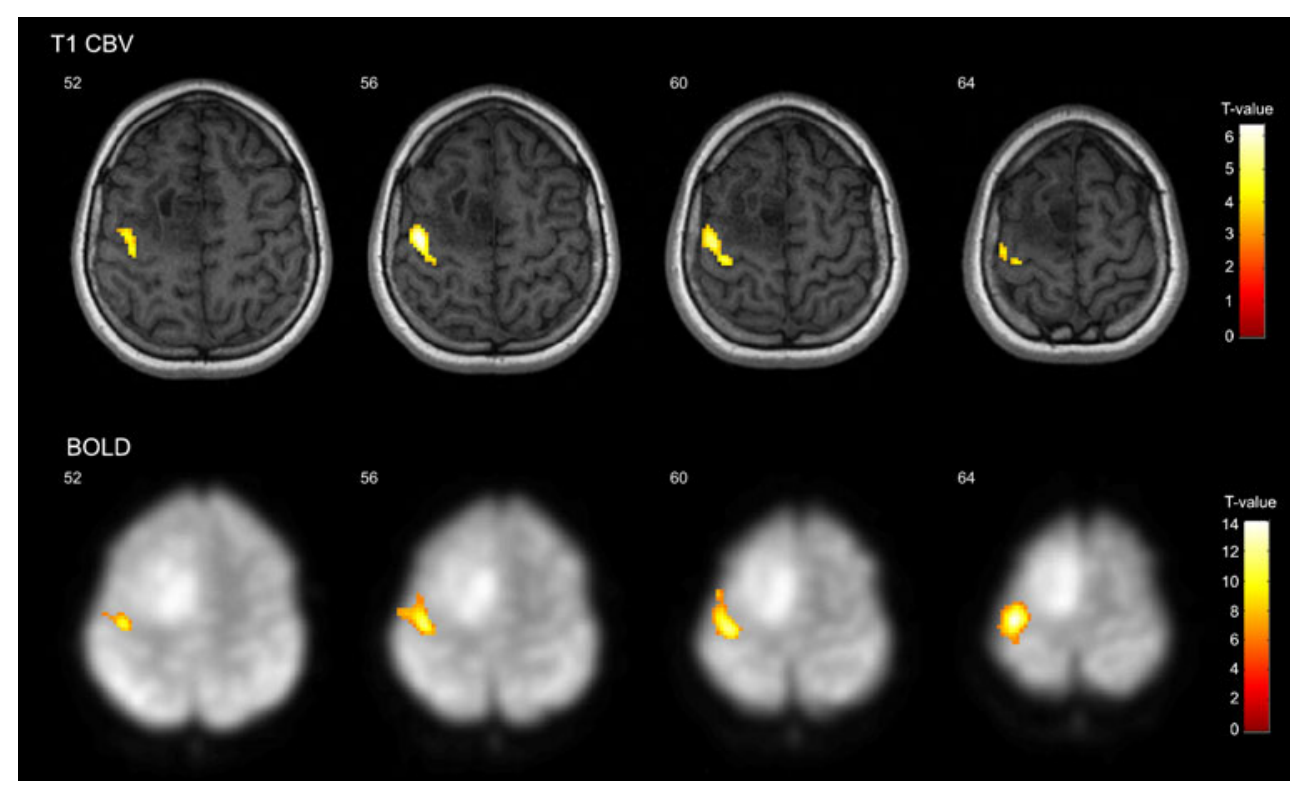




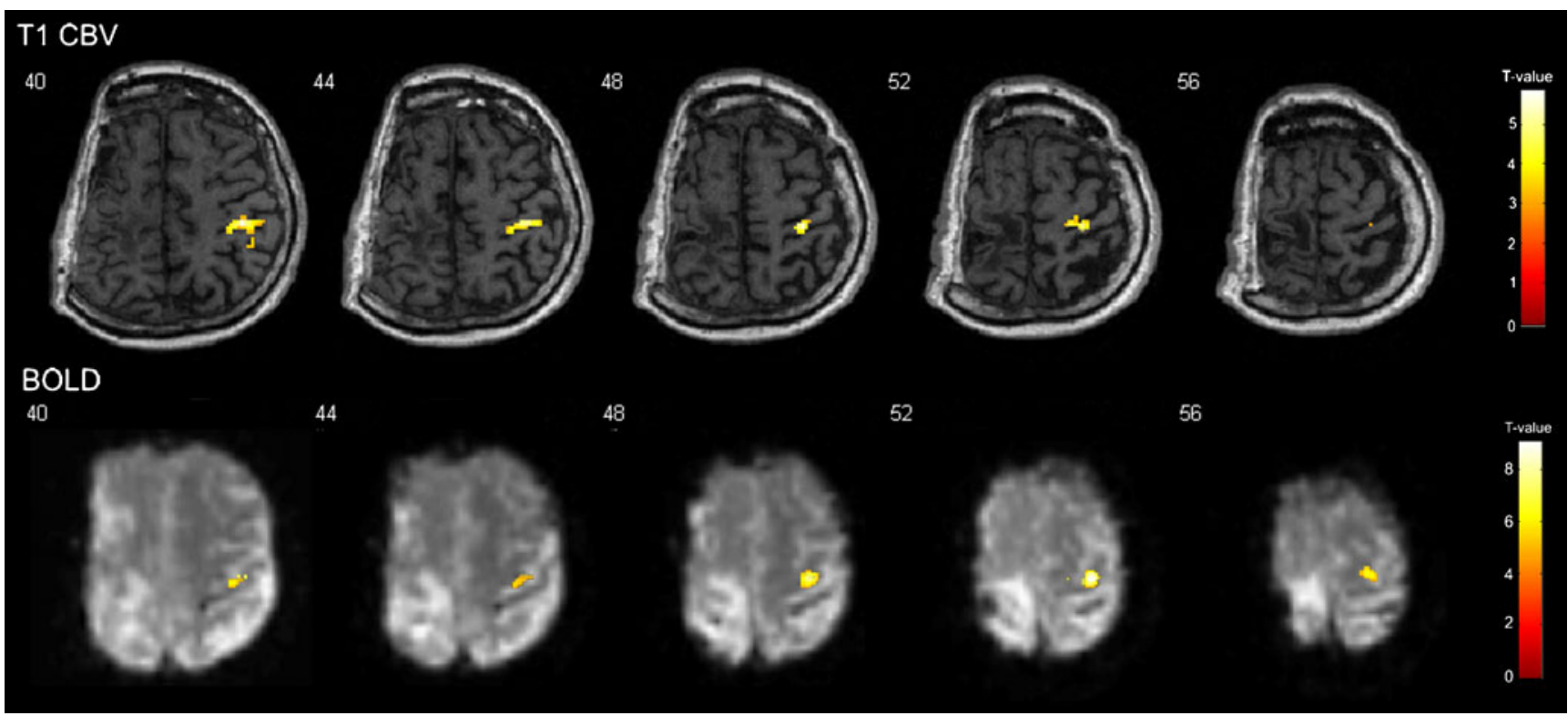

Fig. 2 Patient EF after surgery with encefalomalacia areas in the left precentral gyrus and parietal lobe; activations in PMC in technique T1 CBV upper row, in BOLD technique in lower row during RIGHT hand movement

This was particularly the case of one of our female patients, whose clinical status was poor due to surgery performed 3 days prior to MR examination. Additionally, the duration of the T1 sequence is long and amounts to $10 \mathrm{~min}$ and $31 \mathrm{~s}-$ twice as long as for EPI (5 min $11 \mathrm{~s}$ )-another factor reflected by patient fatigue. Secondly, the risk of movement artifact occurrence elevates significantly with test of such long duration. Thirdly, $t$ values of cortical activities recorded by means of the T1 CBV technique were significantly lower compared to the BOLD contrast method; thus, cortical activity may have been markedly suppressed, even by low-intensity motion artifacts.

Fig. 3 Patient MF after surgery; activations in CBL in T1 CBV technique upper row, in BOLD technique in lower row during right hand movement
Additionally, our experience suggests the necessity for creating a new method or significant modification of existing processing methods of data obtained by means of the T1 CBV technique. While the SPM statistical package is being widely and effectively used in calculations related to T2* BOLD examinations, it has significant limitations in context of its application to T1 CBV results. The volume subjected to analysis is one of the principal differences between these two techniques. In order to accelerate calculations performed with the SPM software, a mask is determined for the points subjected to these calculations. The points whose signal is close to zero are discarded from

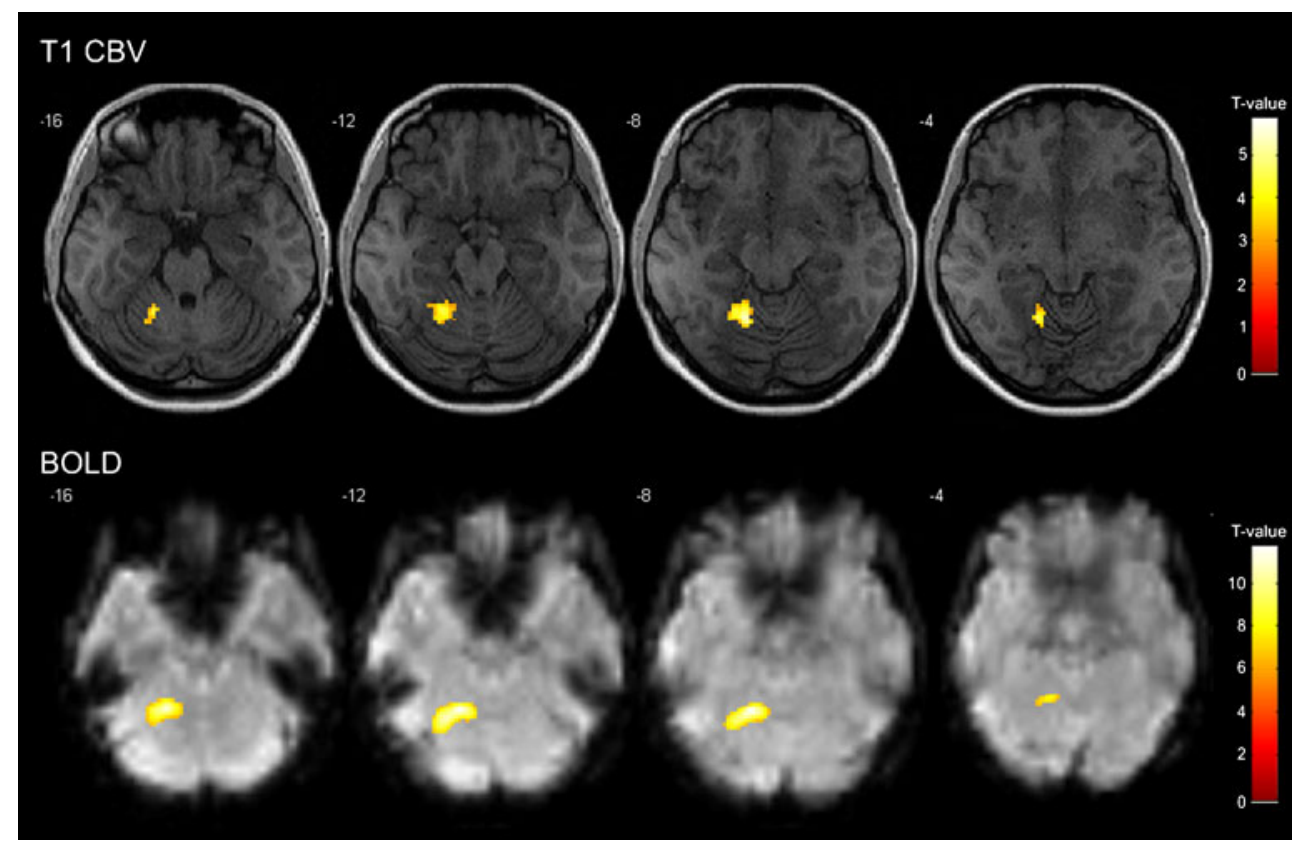




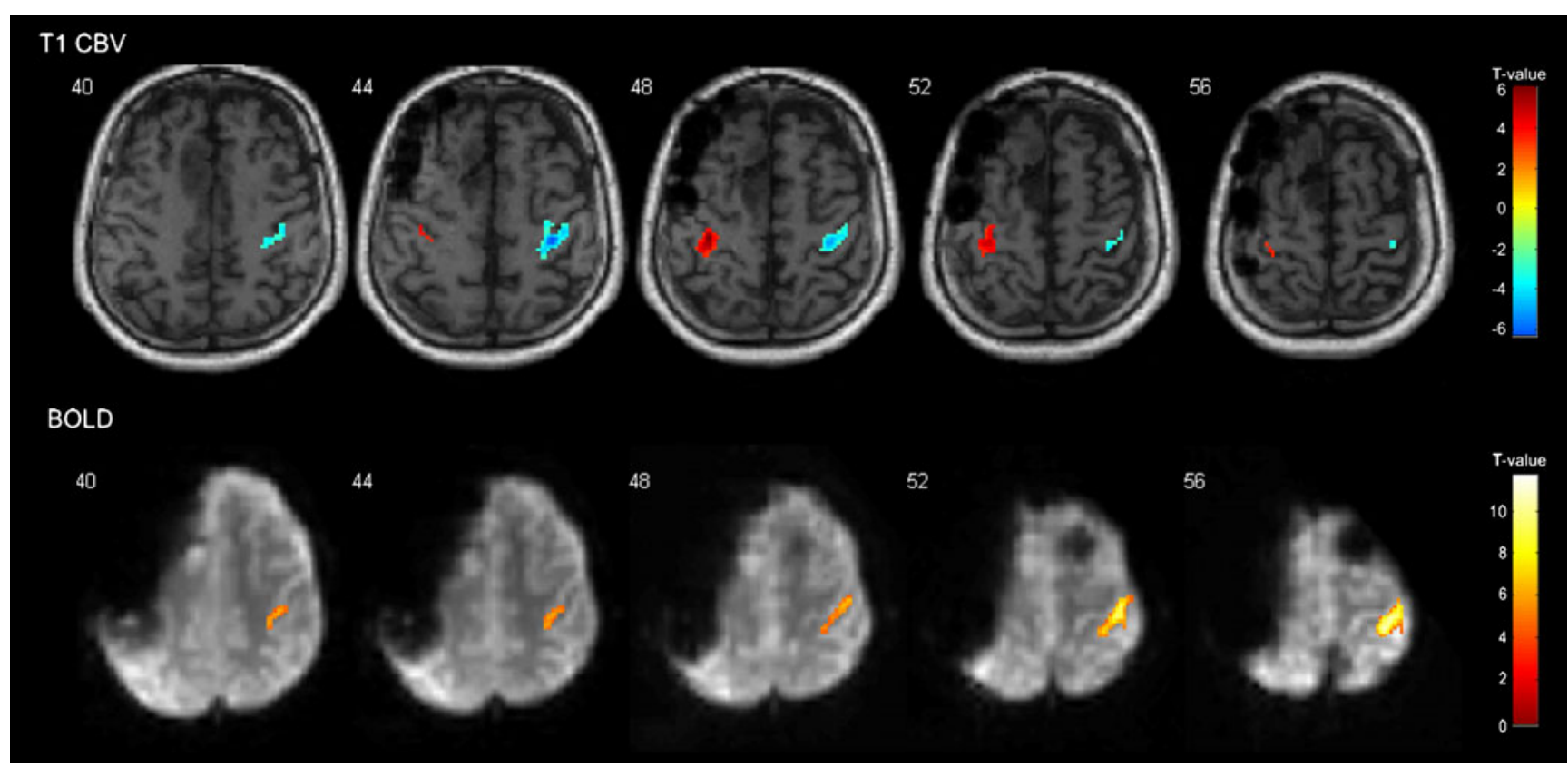

Fig. 4 Patient $\mathrm{BC}$ after surgery with presence of cranio-fixes in the left frontal lobe area; activations in PMC in a T1 CBV techniqueupper row, during right hand movement — red color, during left hand movement-blue color. b in BOLD technique-lower row, during right hand movement - no activation because of vast area of artifacts, during left hand movement-yellow color further analyses. Therefore, only the brain volume is subjected to further analyses in the case of echo planar imaging, compared to nearly the entire head, including bones and skin tissues, in the case of T1 images. Additionally, there is a risk of signal loss when examining the ventricles and deep grooves, where depending on individual morphology, signal intensity may be close to zero.

Along with Morton's findings [15], but opposite to the results published by Ben Bashat et al. [17], we observed that in $83.3 \%$ of cases, $t$ values of activation were lower in the T1 CBV technique. The results of functional T1 CBV examinations are influenced by several factors, including the type of paradigm applied, the intensity of magnetic field, image noise due to voxel size, bandwidth, and type of MR sequence as well as various $t$ effects of head motion [26]. Moreover, the sequences we used differed significantly in terms of their resolution: the voxel sizes were 3.75 and $1.78 \mathrm{~mm}$ for $\mathrm{T}_{2} *$ and $\mathrm{T} 1$ sequences, respectively. The objective of our study was to compare two methods using sequences which are widely available in MR examination rooms. Nonetheless, simultaneously, we continue studies with high-resolution $\mathrm{T} 2 *$ BOLD sequences.

Different ranges of $t$ values for both methods compared in this study seem to have no practical importance. The groups we analyzed were small, particularly in the case of subjects examined preoperatively. The group of subjects after neurosurgeries was inhomogeneous in terms of postsurgical area location, its shape and size, co-existing softening and/or edema, type of surgery, and the presence of potential relapse.

Similar to the BOLD contrast method, the T1 CBV technique's ability to detect activation is not limited to primary sensorimotor centers. In our study, three tasks performed by two subjects after surgeries revealed activation within the cerebellar region as well. Similar to activations observed in superior centers, $t$ values obtained using the CBV technique were lower compared to those revealed by the BOLD contrast method. The possibility of determining functional brain reorganization and plasticity processes in patients subjected to neurosurgeries is particularly important - both to fully understand these mechanisms and in context of planning potential sparing re-surgeries.

Depending on the overall status of the patients, a different number of paradigms, from 1 to 3, was applied both for the EPI and T1 techniques. Clinical application of blood pool contrast agents, probably the first in history, was the factor which enabled performing the entire functional examination procedure, including the required number of paradigms. The blood concentration of these agents is constant and they remain in circulation long enough for standard fMRI procedures, with no need of additional doses added every several minutes. Consequently, it is possible to use additional diagnostic morphological sequences with the same single dose of contrast agent, as was confirmed in our study.

Similar to $\mathrm{T} 2 * \mathrm{BOLD}$, the sensitivity of $\mathrm{T} 1 \mathrm{CBV}$ increased when more than one paradigm was applied [27, 
28]. In one patient (AS), the location of the primary sensorimotor center was established based on increased signal intensity in the tactile paradigm, while no activity of this center was observed during movement task.

The necessity for contrast agent application in T1 CBV is undoubtedly its limitation compared to $\mathrm{T} 2 * \mathrm{BOLD}$, making the former technique an invasive one. Nonetheless, T1 CBV may be applied in cases where there are clinical indications for the use of contrast agents, with suspicion of tumor relapse being one of the cases where the use of contrast agents is obligatory. In this study, we used Vasovist agent at a dose recommended by its manufacturer for standard examinations - MR angiography of abdominal and lower limb vessels-i.e., $0.12 \mathrm{ml} / \mathrm{kg}$ b.w., which corresponds to $0.03 \mathrm{mmol} / \mathrm{kg} \mathrm{b}$.w. Further research is needed to determine if this dosage requires optimization in order to improve signal acquisition during functional studies.

Contrary to Ben Bashat et al., we did not observe activation around neoplastic tumors using the T1 CBV technique. According to these authors, activation observed around the tumor on T1 CBV images would suggest that evaluation of angiogenesis is possible using this method [17]. This would be another potential interesting application of this new technique, but further research is needed to optimize both the T1-weighted sequences dedicated to functional examination and the distribution profile of the contrast agent. As for the lack of activation around tumors found in our study, this might be related to differences in the microscopic structure of neoplasms we examined compared to those analyzed by Ben Bashat et al. [17].

\section{Conclusions}

In conclusion, the $\mathrm{T} 1 \mathrm{CBV}$ technique can be used to detect functional changes in the sensorimotor cortex of patients with brain tumors, both before and after surgical intervention. The centers of activation in both the BOLD and T1 CBV techniques have the same localization; however, the increase in signal intensity was lower for the T1 CBV technique. Therefore, this technique is more sensitive to movement artifacts. The T1 CBV technique enables accurate evaluation of cortical centers, which is usually disturbed when using the BOLD method due to magnetic susceptibility artifacts. Clinical application of the T1 CBV technique requires improved methods of data collection and analysis, as well as determination of correct dosages of contrast agent.

Conflict of Interest We declare that we have no conflict of interest.

Open Access This article is distributed under the terms of the Creative Commons Attribution Noncommercial License which permits any noncommercial use, distribution, and reproduction in any medium, provided the original author(s) and source are credited.

\section{References}

1. Voyvodic JT, Petrella JR, Friedman AH (2009) fMRI activation mapping as a percentage of local excitation: consistent presurgical motor maps without threshold adjustment. J Magn Reson Imaging 29:751-759

2. Ramsey NF, Hoogduin H, Jansma JM (2002) Functional MRI experiments: acquisition, analysis and interpretation of data. Eur Neuropsychopharmacol 12:517-526

3. Lehéricy S, Duffau H, Cornu P et al (2000) Correspondence between functional magnetic resonance imaging somatotopy and individual brain anatomy of the central region: comparison with intraoperative stimulation in patients with brain tumors. J Neurosurg 92:589-598

4. Stippich C (2007) Presurgical functional Magnetic Resonance Imaging (fMRI). Clin Neuroradiol 17:69-87

5. Deichmann R, Josephs O, Hutton C, Corfield DR, Turner R (2002) Compensation of susceptibility-induced BOLD sensitivity losses in echo-planar fMRI imaging. Neuroimage 15:120-135

6. Gorno-Tempini ML, Hutton C, Josephs O, Deichmann R, Price C, Turner R (2002) Echo time dependence of BOLD contrast and susceptibility artifacts. Neuroimage 15:136-142

7. Weiskopf N, Hutton C, Josephs O, Deichmann R (2006) Optimal EPI parameters for reduction of susceptibility-induced BOLD sensitivity losses: a whole-brain analysis at $3 \mathrm{~T}$ and $1.5 \mathrm{~T}$. Neuroimage 33:493-504

8. Truong TK, Clymer BD, Chakeres DW, Schmalbrock P (2002) Three-dimensional numerical simulations of susceptibilityinduced magnetic field inhomogeneities in the human head. Magn Reson Imaging 20:759-770

9. Dubowitz DJ, Bernheim KA, Chen DY, Bradley WG Jr, Andersen RA (2001) Enhancing fMRI contrast in awake-behaving primates using intravascular magnetite dextran nanopartieles. NeuroReport 12:2335-2340

10. Smirnakis SM, Schmid MC, Weber B, Tolias AS, Augath M, Logothetis NK (2007) Spatial specificity of BOLD versus cerebral blood volume fMRI for mapping cortical organization. Cereb Blood Flow Metab 27:1248-1261

11. Keilholz SD, Silva AC, Raman M, Merkle H, Koretsky AP (2006) BOLD and CBV weighted functional magnetic resonance imaging of the rat somatosensory system. Magn Reson Med 55:316-324

12. Zhao F, Jin T, Wang P, Hu X, Kim SG (2007) Sources of phase changes in BOLD and CBV-weighted fMRI. Magn Reson Med 57:520-527

13. Mandeville B, Marota J, Keltner JR, Weissleder R, Rosen B, Weisskoff R (1998) Dynamic functional imaging of relative cerebral blood volume during rat forepaw stimulation. Magn Reson Med 39:615-624

14. Marota JJ, Ayata C, Moskowitz MA, Weisskoff RM, Rosen BR, Mandeville JB (1999) Investigation of the early response to rat forepaw stimulation. Magn Reson Med 41:247-252

15. Morton DW, Keogh B, Lim K, Maravilla KR (2006) Functional brain imaging using a long intravenous half-life gadolinium-based contrast agent. AJNR Am J Neuroradiol 27:1467-1471

16. Majos A, Bogorodzki P, Piatkowska-Janko E, Wolak T, Kurjata R, Stefańczyk L (2009) Functional imaging with MR T1 contrast: a feasibility study with blood-pool contrast agent. Eur Radiol 19:898-903

17. Ben Bashat D, Sivan I, Ziv M, Aizenstein O, Pianka P, Malach R, Graif M, Hendler T, Navon G (2008) T1-weighted functional imaging based on a contrast agent in presurgical mapping. J Magn Reson Imaging 28:1245-1250

18. Niemi P, Koskinen S, Reisto T (1991) Tissue relaxation enhancement after intravenous administration of (ITCB-DTPA)-gadolinum conjugated albumin, an intravascular magnetic resonance imaging contrast agent. Invest Radiol 26:674-680 
19. Parmelee DJ, Walovitch RC, Ouellet HS, Lauffer RB (1997) Preclinical evaluation of the pharmacokinetics, biodistribution, and elimination of MS-325, a blood pool agent for magnetic resonance imaging. Invest Radiol 32:741-747

20. Rohrer M, Bauer H, Mintorovitch J, Requardt M, Weinmann HJ (2005) Comparison of magnetic properties of MRI contrast media solutions at different magnetic field strengths. Invest Radiol 40:715-724

21. Giovagnoni A, Catalano C (2007) Application of blood-pool agents in visualization of peripheral vessels. Eur Radiol 17(Suppl 2):B18-B23

22. Caravan P, Parigi G, Chasse JM, Cloutier NJ, Ellison JJ, Lauffer RB, Luchinat C, McDermid SA, Spiller M, McMurry TJ (2007) Albumin binding, relaxivity, and water exchange kinetics of the diastereoisomers of MS-325, a gadolinium(III)-based magnetic resonance angiography contrast agent. Inorg Chem 46:6632-6639

23. Essig M, Rohrer M, Giesel F, Tüttenberg J, Weber MA, Michaely H, Gerigk L, Voth M (2010) Human brain tumor imaging with a proteinbinding MR contrast agent: initial experience. Eur Radiol 20:218-226
24. Shin W, Gu H, Yang Y (2009) Fast high-resolution T1 mapping using inversion-recovery Look-Locker echo-planar imaging at steady state: optimization for accuracy and reliability. Magn Reson Med 61:899-906

25. Stehling MK, Ordidge RJ, Coxon R, Mansfield P (1990) Inversion-recovery echo-planar imaging (IR-EPI) at $0.5 \mathrm{~T}$. Magn Reson Med 13:514-517

26. Smirnakis SM, Schmid MC, Weber B, Tolias AS, Augath M, Logothetis NK (2007) Spatial specificity of BOLD versus cerebral blood volume fMRI for mapping cortical organization. J Cereb Blood Flow Metab 27:1248-1261

27. Stippich C, Heiland S, Tronnier V, Mohr A, Sartor K (2002) Functional magnetic resonance imaging: Physiological background, technical aspects and prerequisites for clinical use. Rofo 174:43-49

28. Rossini PM, Dal Forno G (2004) Integrated technology for evaluation of brain function and neural plasticity. Phys Med Rehabil Clin N Am 15:263-306 